\title{
Die Modularisierung der Berufsausbildung ist ein Fehlkonzept
}

\author{
GEORG SPÖTTL
}

\section{Beruflichkeit oder Modularisierung?}

Seit den 1990er Jahren wird die Frage nach einer Modularisierung der Berufsbildung diskutiert, wobei es hierbei nicht nur um unterschiedliche Ansätze, sondern auch um sehr verschiedene Grundstrukturen von Berufsbildung geht. Es steht die Auseinandersetzung an zwischen

- einem Berufsbildungssystem, ${ }^{2}$ basierend auf Berufen und

- einem Qualifizierungskonzept, basierend auf flexibel organisierbaren Modulen.

Im ersten Fall steht die Entwicklung der sogenannten umfassenden beruflichen Handlungskompetenz und der beruflichen Identität im Mittelpunkt; im zweiten Fall wird in abgegrenzten Einheiten gelernt und die Qualität der Lernergebnisse durch vom Lernen losgelöste Tests (so der anglo-amerikanische Ansatz) festgestellt. Ziel ist dabei vermeintlich die direkte Qualifizierung für Aufgaben in der Arbeitswelt.

Auslöser für eine intensive Richtungsdiskussion waren unter anderem Positionierungen des Europäischen Parlaments ${ }^{3}$ mit dem Ziel, die transnationale berufliche Mobilität der Beschäftigten und Lernenden über lebenslanges Lernen zu ermöglichen und dazu beizutragen, den Anforderungen von Angebot und Nachfrage des europäischen Arbeitsmarktes zu entsprechen. Vereinfacht betrachtet wurde von zahlreichen Akteuren, von Politikern, von Berufsbildungsplanern und von Interessenvertretern verschiedenster Richtungen der Schluss gezogen, dass definierte Zielsetzungen mit Modulstrukturen deutlich leichter erreichbar seien, als dieses auf der Basis von Berufsstrukturen möglich sei. Berufe und ein Berufsbildungssystem, wie sie in Deutschland für die berufliche Erstausbildung existieren, wurden als eher hinderlich betrachtet. Stattdessen wurden Qualifizierungskonzepte basierend auf Modulen hoch favorisiert und als Instrumente gewertet, mit denen (endlich) flexible Ausbildungs- und Qualifizierungsprozesse implementiert werden können.
Zudem wurden und werden mit Blick auf das duale System Hoffnungen verbunden, diesem durch Modularisierung mehr Beweglichkeit einhauchen zu können. Damit mutieren jedoch Modularisierungsüberlegungen in der Regel schnell zu einem organisatorischen Instrument, das Flexibilität in der Anordnung von ,abgegrenzten und zertifizierten Lerneinheiten" schaffen will, aber damit noch lange keine umfassende Kompetenz in der Ausbildung garantiert. Eher das Gegenteil ist der Fall, weil abgegrenzte Lerneinheiten nur eine fragmentierte Qualifizierung erlauben.

Grundlage der verschiedenen Überlegungen zur Modularisierung ist die Idee, dass Berufsqualifikationen aus einer Kombination von Teilqualifikationen bestehen, durch deren Akkumulation die Gesamtfunktion, also die berufliche Handlungskompetenz, erreicht wird. Dabei verhindert die Dominanz, etwas zu organisieren, lerntheoretische und didaktische Überlegungen einzubringen, um eine erfolgreiche Kompetenzentwicklung zu garantieren. Zudem werden der Arbeitsprozessbezug und der Aufbau einer beruflichen Identität nicht oder nur am Rande in die Überlegungen einbezogen, weil Modularisierungsansätze meist auf kurzfristig angelegte Trainingsmaßnahmen und nicht auf längerfristige Kompetenzentwicklung angelegt sind.

Der Kerngedanke der Modularisierung, nämlich mehr Flexibilität mit Blick auf das Gelernte

\footnotetext{
Meist wird darunter eine in sich abgeschlossene Einheit verstanden, auf Grundlage derer beispielsweise gelernt werden kann.

(2) Vgl. Kurtz, Th. (2005): Die Berufsform der Gesellschaft, Weilerwist.

(3) Vgl. Europäisches Parlament (2007): Standpunkt des Europäischen Parlaments festgelegt in erster Lesung am 24. Oktober, Brüssel.

(4) Vgl. Bünning, F./Hortsch, H./Novy, K. (2000): Das britische Modell der National Vocational Qualifications (NVQs). Ausgangspunkt für eine Modularisierung beruflicher Bildung in Deutschland, Hamburg.
}

zu erreichen, bleibt demnach eine reine Behauptung. Eine Analyse der detaillierten, auf kleinste Module bezogenen Zertifizierungskonzepte in englischsprachigen und asiatischen Ländern belegt vielmehr eine Behinderung von Flexibilität durch extremen Organisationsaufwand und „Anpassungsqualifizierung“· nach eng ausgerichteten Modulen bedeutet ein Abrichten auf bestimmte Tätigkeiten. Die Absicht dabei ist, ein „Matching“ zu ermöglichen, also eine genaue Deckung zwischen Arbeitstätigkeit und Modulinhalt zu erreichen, um dadurch die Beschäftigungsfähigkeit zu garantieren. In Ländern mit modularisierten Strukturen gelingt es allerdings nicht, diese Deckung zu bewerkstelligen. Die Tätigkeiten sind zu vielfältig und verändern sich zu schnell, um diese über Modul- und Zertifizierungsstrukturen abbilden zu können. Wird die viel beschworene Passung nicht erreicht, führt das oft zu Arbeitslosigkeit der Jugendlichen und Erwachsenen ${ }^{\ominus}$ oder bei günstiger wirtschaftlicher Entwicklung zu der Notwendigkeit von intensiven Nachqualifizierungen durch Industrie und andere Arbeitgeber. Dieses dokumentiert sich dann in einer unübersichtlichen Vielzahl an Angeboten von Trainingsund Qualifizierungsinstituten. Modularisierung ist kein Garant für Beschäftigungsfähigkeit, eher wird Beschäftigungsfähigkeit durch Modularisierung behindert. 
Eine weitere, oft bemühte Begründung für Modularisierung ist die Tatsache, dass sich die Stabilität von Erwerbsverläufen von der Ausbildungsphase bis ins spätere Alter deutlich gewandelt hat. Unsicherheiten und Verwerfungen in der Erwerbsbiografie in einer global ausgerichteten Produktion bestehen über das gesamte Erwerbsleben hinweg, was zu veränderten Anforderungen in der Anpassungs- und Neugestaltungsfähigkeit führt. Untersuchungen von Bolder ${ }^{\bullet}$ zeigen allerdings, dass dieser Wandel nicht in der oft geschilderten Dramatik und Geschwindigkeit vonstattengeht, sondern die Veränderungen über einen sehr langen Zeitraum stattfinden und Kontinuität eine große Rolle spielt.

Auffällig ist inzwischen, dass in den beruflichen Schulen Modularisierung mit Blick auf die geforderten Klassengrößen als geeigneter Ansatz diskutiert wird, um die vorgegebene Anzahl von wenigstens 15 Schülern je Klasse zu erreichen. Aufgrund der hohen Zahl schwach besetzter Ausbildungsberufe gelingt Letzteres den Schulen immer weniger. Um dieses Problem zu lösen, gehen die Berufsschulen dazu über, Schüler aus verschiedenen Jahrgängen gemeinsam zu unterrichten. Das bedeutet, dass Lehr-/Lerneinheiten modular zusammengestellt werden, damit ein jahrgangsübergreifender Unterricht möglich wird. Dafür werden Inhalte ausgewählt, die vermeintlich eigenständig gelehrt werden können und sich für unterschiedliche Zielgruppen eignen sollen. Deutlich wird: Hier dominiert der organisatorische Aspekt, um zielgruppenübergreifende Kompetenzentwicklungsangebote garantieren zu können.

\section{Modularisierung - ein Instrument für den Umbau des Berufsbildungssystems?}

Befürworter einer Modularisierung kritisieren drei Fehlentwicklungen im derzeitigen Berufsbildungssystem:

(1) Im dualen System werden immer weniger Hauptschüler und andere schwächere Schulabsolventen aufgenommen, sodass dieses zum „goldenen Weg“ für die „Stärkeren“ geworden ist.

(2) Mehr als 400.000 Jugendliche und junge Erwachsene halten sich in Übergangsmaßnahmen, Warteschleifen oder Arbeitslosigkeit auf und haben keine oder nur geringe Aussichten auf Ausbildungsverträge im dualen System.

(3) Die Zahl der Ausbildungsverträge im dualen System geht seit drei Dekaden kontinuierlich zurück, weil Betriebe immer weniger ausbilden. ${ }^{\circ}$
Kritiker einer Modularisierung stellen in erster Linie fest:

(1) Gestaltungsorientierte Lern- und Entwicklungsprozesse sind gefährdet, weil es bei Modulen um eine flexible Organisation von Konzepten und nicht um eine Systemorganisation von Lernen geht.

(2) Inhalte werden in Komponenten zerlegt und stehen isoliert nebeneinander, auch wenn sie lerntheoretisch gesehen gemeinsam unterrichtet werden müssen. Damit wird die Gefahr der beliebigen Austauschbarkeit von Lerninhalten erhöht.

(3) Noch desorientierte, unterqualifizierte Jugendliche werden durch die Modularisierung verstärkt einem selektiven, deregulierten Ausbildungsmarkt ohne pädagogischen Schutzraum überlassen.

(4) Bei modularisierten Konzepten ist es nicht möglich, arbeitsprozessbasierten Ansätzen zu folgen, weil sich die klar umrissenen Modulinhalte per Struktur dem Prozessgedanken verschließen, der jedoch für eine berufliche Ausbildung in Bezug auf komplexere Tätigkeiten in Produktion und Dienstleistung unabdingbar zu berücksichtigen ist.

(5) Modularisierte Konzepte verfolgen keine berufliche Handlungskompetenz, sondern sie zielen auf die Zertifizierung kleiner, abgrenzbarer Lerneinheiten, mit der Priorität, dass deren Lernergebnisse eindeutig definierbar und prüfbar sind.

(6) Modularisierung und Beruflichkeit sind ein Widerspruch in sich, weil Module auf sachlich abgegrenzte Einheiten zielen, während Beruflichkeit umfassende Handlungskompetenz und Identitätsbildung zum Ziel hat.

Die Für- und Wider-Argumente belegen deutlich, dass im Hintergrund der Modularisierungsdiskussion die Frage nach dem Erhalt des derzeitigen Ausbildungssystems mit beruflicher Ausrichtung steht oder die alternative Orientierung auf flexible Organisationskonzepte mit angepasster Qualifizierung für den Arbeitsmarkt. Vereinbar sind die beiden Ansätze aufgrund ihrer Gegensätze nicht: Mit Blick auf Kompetenzentwicklung geht es in der dual organisierten Berufsbildung um die Entwicklung von beruflicher Handlungskompetenz, wobei curricular organisierte Lernprozesse eine wichtige Rolle spielen. Modularisierungsansätze haben dagegen das Ziel, erworbene Qualifikationen direkt verwertbar zu machen.

\section{Grenzen der Modularisierung}

In der Modularisierungsdiskussion wird meist übersehen, dass die im dualen System verankerte Beruflichkeit und Handlungskompetenz mehr ist als nur ein Fertigkeits- und Fähigkeitstraining. Vielmehr wird durch die Heranführung der Jugendlichen an die betrieblichen und gesellschaftlichen Grundstrukturen wesentlich zur Legitimation des vorherrschenden Systems der Berufsausbildung beigetragen. Dies steht in ganz enger Verbindung mit der Entwicklung beruflicher Identität. Durch diese Ausrichtung werden die Jugendlichen nicht nur für berufliche und betriebliche Aufgaben befähigt, sondern auch zur Wahrnehmung und Mitgestaltung gesellschaftsrelevanter Anliegen.

Dem wird in der Regel entgegengestellt, dass eine dynamische Arbeitswelt höchste Anforderungen an die Flexibilität der Beschäftigten stellt, die bei einer Beruflichkeitsorientierung nicht gewährleistet sei.

Hier liegt ein großes Missverständnis vor. Beruflichkeit bedeutet ganz und gar nicht, dass die Flexibilität eingeschränkt oder im Kern der Ausbildung nicht angelegt wird. Ein genauerer Blick in die Ausbildungsordnungen zeigt, dass dort die Bewältigung neuer Arbeitsanforderungen, die Entwicklung von Kreativität, von Lernbereitschaft und Problemlösefähigkeit sowohl mit berufsbezogenen als auch mit berufsübergreifenden Dimensionen genannt ist. Das garantiert berufliche Flexibilität, die auch wahrgenommen wird, wie wissenschaftliche Untersuchungen belegen. ${ }^{8}$

Trotzdem sehen Akteure in der Umgestaltung vor allem der dualen Berufsausbildung hin zu einem modularen Konzept wichtige Schritte, um den europäischen Vorstellungen näher zu

6 Bolder, A. (2007): Erwerbsbiographien im Wandel - Konsequenzen für die Berufsbildung, in: Kuda, E. (Hrsg.): Fachlich kompetente Arbeit in Europa - Neue Wege in der beruflichen Bildung, Expertenworkshop, IG Metall Vorstand, Frankfurt a. M., S. 22-33.

$(7$ Vgl. hierzu Hucker, T. (2012): Betriebliche Ausbildungsbeteiligung stark rückläufig, in: Berufsbildung in: Wissenschaft und Praxis (BWP) (4), S. 4-5.

8 Hall, A. (2007): Beruflichkeit: Fundament oder Hindernis für Flexibilität? Berufswechsel von dual ausgebildeten Fachkräften, in: BWP (4), S. 10-14; Spöttl, G./Windelband, L. (2012): Arbeitsprozessorientierte Berufsbildung im dualen System - sechs Innovationen zur Zukunftssicherung (Teil 1), in: Die berufsbildende Schule (BbSch) 64 (9), S. 255-259. 
kommen. Die Spitze des Eisberges dieser Diskussion dürfte die Auseinandersetzung um Ausbildungsbausteine sein, die durch das Gutachten von Euler und Severing angestoßen wurde ${ }^{\odot}$ und ihre Fortsetzung in der Gestaltung der Ordnungsmittel findet. ${ }^{(1)}$

Die Debatte wird von Vorschlägen beherrscht, die die Auflösung von Ausbildungsgängen zugunsten von Teilqualifikationen im Zentrum hat. Das dafür zweifelsohne am häufigsten genutzte unterstützende, aber unreflektierte Argument ist, mehr Flexibilität in der Ausbildung zu erreichen. Tatsächlich aber zeigt sich, dass der Besitz nur von Teilqualifikationen Flexibilität behindert, weil die so ausgebildeten Personen nur sehr begrenzt einsetzbar sind. Bei der Evaluation des zweijährigen Kfz-Servicemechanikers-Ausbildungsganges konnte beispielsweise belegt werden, dass nach dieser verkürzten Ausbildung nur rund $11 \%$ der Absolventen einen Arbeitsvertrag erhalten haben. ${ }^{(1)}$ Als Begründung für die Nichteinstellung wurde von den Unternehmen mit Abstand am häufigsten genannt, dass es den Absolventen an der Fähigkeit fehlen würde, umfassende Arbeiten an Fahrzeugen durchführen zu können.

\section{Schlussfolgerungen}

Die Vorschläge zur Modularisierung lassen sich in der These zusammenfassen, dass es dabei in erster Linie um Konzepte geht, wonach sich Lerneinheiten effektiver organisieren lassen. Die Gestaltung erfolgreicher Lernprozesse nah an den beruflichen Arbeitsprozessen, die im Ergebnis zur beruflichen Arbeit befähigen und berufliche Flexibilität erhöhen, spielt keine Rolle. Berufliche Handlungskompetenz und Identitätsbildung sowie grundlegende, qualifizierende und sozialisierende Berufsausbildung werden durch Modularisierung zugunsten einer verkürzten Beschäftigungsorientierung mittels Anpassungstraining verdrängt. Indikatoren, dass Modularisierung die Chancen von Jugendlichen auf dem Ausbildungsmarkt verbessert, sind nicht identifizierbar. Die immer wieder ins Feld geführte Flexibilisierung der Ausbildung kann gerade durch Modularisierungskonzepte nicht erreicht

(9) Euler, D./Severing, E. (2006): Flexible Ausbildungswege in der Berufsbildung, Typoskript, Nürnberg/St. Gallen; Dies. (2007): Flexible Ausbildungswege in der Berufsbildung, Bielefeld.

(10) Vgl. hierzu Frank, J. (2012): Start frei für kompetenzorientierte Gestaltung der Ordnungsmittel? Konsequenzen des DQR für die Ordnungsarbeit, in: BWP (4), S. 49-52.

(11) Vgl. Becker, M./Musekamp, F./Spöttl, G. (2012): Zweijährige Ausbildung zum Kfz-Servicemechaniker - ein Beruf mit Zukunftschancen?, in: Die berufsbildende Schule (BbSch) 64 (1), S. 18-26. werden. Sie kann dagegen für eine als System organisierte duale Berufsbildung in Anspruch genommen werden. Gegenüber modularisierten Ansätzen, die oft mit vielfältigen Ausstiegsoptionen verbunden sind, hat der systembezogene Beruflichkeitsansatz mit Ausrichtung auf Arbeitsprozesse eindeutig den Vorteil, dass für die Wahrnehmung umfassender beruflicher Aufgaben qualifiziert wird. Dadurch verbessern sich die Einstiegschancen in das Erwerbsleben und es wird hohe Flexibilität im innerbetrieblichen und überbetrieblichen Einsatz gewährleistet. Aus dieser knappen Analyse heraus kann den Gestaltern des deutschen Berufsbildungssystems nur empfohlen werden, es nicht auf dem Altar der Modularisierung zu opfern.

\section{AUTOR}

GEORG SPÖTTL, Prof. Dr., ist Vorstandsmitglied des Instituts Technik und Bildung (ITB) an der Universität Bremen. Arbeitsschwerpunkte: Berufswissenschaftliche Qualifikationsforschung, Curriculumentwicklung, internationale Berufsbildung.

spoettl@uni-bremen.de 